\title{
BIO-ANALYTICAL METHOD DEVELOPMENT AND VALIDATION FOR THE SIMULTANEOUS ESTIMATION OF DECITABINE AND CEDAZURIDINE IN HUMAN PLASMA USING LC-MS/MS
}

\section{SAI PRUDHVI N. ${ }^{1,2^{*}}$, VENKATESWARLU B. S. ${ }^{3}$, KUMUDHAVALLI M. V. ${ }^{4}$, MURUGANANTHAM V. ${ }^{3}$}

${ }^{1}$ Vinayaka Missions College of Pharmacy, Salem, India, ${ }^{2}$ Department of Pharmaceutical Analysis, M. A. M. College of Pharmacy, Guntur, India, ${ }^{3}$ Department of Pharmaceutics, Vinayaka Missions College of Pharmacy, Salem, India, ${ }^{4}$ Department of Pharmaceutical Analysis, Vinayaka Missions College of Pharmacy, Salem, India *Email: saiprudhvi00@gmail.com

Received: 09 Apr 2021, Revised and Accepted: 11 Jun 2021

\section{ABSTRACT}

Objective: The present work aimed to develop a novel, reliable and accurate Liquid Chromatography-Mass Spectrometry/Mass spectrometry (LCMS/MS) method for the simultaneous quantification of Decitabine and Cedazuridine a combined medication used for the treatment of chronic myelomonocytic leukemia in human plasma.

Methods: Talazoparib drug is used as an internal standard in the study. Both the analytes and internal standard were isolated from $100 \mathrm{ml}$ plasma samples by liquid-liquid extraction and then chromatographed on Zorbax SB-CN ( $4.6 \mathrm{~mm} \times 75 \mathrm{~mm}, 3.5 \mu \mathrm{m})$ column with a mobile phase consisting of 0.1 $\%$ ammonium formate and methanol in the ratio of $65: 45(\mathrm{v} / \mathrm{v})$ pumped at $0.5 \mathrm{ml} / \mathrm{min}$. The method had a chromatographic total run time of $5 \mathrm{~min}$.

Results: The developed method gave a symmetric peak at a retention time of 1.7 min for Decitabine, 2.2 min for Cedazuridine, 3.5 min for Talazoparib and satisfied all the peak properties as per USP guidelines. The mass spectral characterization of separated analytes in the LC method was performed using a mass detector operated at Multiple Reaction Monitoring mode with precursor-to-product ion transitions at $\mathrm{m} / \mathrm{z}$ of 229 to $\mathrm{m} / \mathrm{z}$ of 114 as $\mathrm{MH}^{+}$ion for Decitabine, $\mathrm{m} / \mathrm{z}$ of 269 to $\mathrm{m} / \mathrm{z}$ of 118 as $\mathrm{MH}^{+}$ion for Cedazuridine. A very sensitive limit of detection of $0.3 \mathrm{ng} / \mathrm{ml}$ was observed and showed a calibration curve linear over the concentration range of LLOQ (lower limit of quantification) to $500 \mathrm{ng} / \mathrm{ml}$. The other validation parameters were found to have acceptable accuracy, precision, linearity, and selectivity. The mean extraction concentration was acceptable and very high for both the analytes in HQC (high-quality control concentration), MQC (medium quality control concentration) and LLOQ levels. The peak area response ratio of Decitabine and Cedazuridine with the internal standard in freeze-thaw, short term and long term stability studies was found to be acceptable confirms that the method is stable.

Conclusion: It can be concluded that the proposed method is specific, accurate, and precise and could be used for the simultaneous estimation of Decitabine and Cedazuridine in human plasma.

Keywords: Decitabine, Cedazuridine, LCMS analysis, Human plasma, Bio-analytical method

(C) 2021 The Authors. Published by Innovare Academic Sciences Pvt Ltd. This is an open access article under the CC BY license (https://creativecommons.org/licenses/by/4.0/) DOI: https://dx.doi.org/10.22159/ijap.2021v13i5.41744. Journal homepage: https://innovareacademics.in/journals/index.php/ijap

\section{INTRODUCTION}

Decitabine (fig. 1a) is a Cytidine analog and nucleic acid synthesis inhibitor drug prescribed for the treatment in dysfunction of certain blood cells (myelodysplastic syndromes) and to treat acute myeloid leukemia [1]. It is structurally related to azacitidine [2]. It is also having significant activity in the treatment of chronic myeloid leukemia [3]. Dose-dependent myelosuppression is the only possible adverse effect while using Decitabine. Nephrotoxicity (nervous system effects) and cardiovascular effects (acute heart failure, myocardial infarction) is the rare side effects observed while using Decitabine.

Cedazuridine (fig. 1b) is a synthetic cytidine deaminase inhibitor and tetrahydrouridine derived nucleoside analogue drug used for the prevention of cytidine breakdown. It is a cytidine deaminase inhibitor that works by binding the enzyme found in gastrointestinal tract and liver and catalyses the deamination of cytidine and cytidine analogues.<smiles>N=C1Cc2nc(=N)nc(O)n2C=N1</smiles>

a) Decitabine

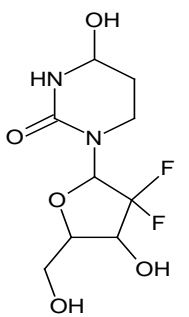

b) Cedazuridine
Fig. 1: Molecular structure of decitabine and cedazuridine
Decitabine and Cedazuridine are the fixed combination medication used for the treatment of myelodysplastic syndromes for both previously treated and untreated patients and for the treatment of chronic myelomonocytic leukemia [4, 5]. Constipation, muscle pain, mucositis, fatigue, joint pain, diarrhea, rash, dizziness, headache, cough and decreased appetite are the possible side effects associated with the use of Decitabine and Cedazuridine combined medication [6].

The literature survey on the available analytical method for the simultaneous analysis of Decitabine and Cedazuridine confirms that only one HPLC method was reported for assay of Decitabine and Cedazuridine in formulations [7]. Few HPLC analytical methods are available for the estimation of Decitabine in formulations [8-11]. Hence the present work aimed to develop a simple and stable LCMS/MS method for the simultaneous estimation of Decitabine and Cedazuridine in human plasma. The Decitabine and Cedazuridine combine medication used for the treatment of chronic myelomonocytic leukemia, another advanced breast cancer treatment drug Talazoparib ((fig. 2) was selected as an internal standard in the study.<smiles>Cn1ncnc1[C@H]1c2n[nH]c(=O)c3cc(F)cc(c23)N[C@H]1c1ccc(F)cc1</smiles>

Fig. 2: Molecular structure of talazoparib (Internal standard) 


\section{MATERIALS AND METHODS}

\section{Equipment and materials}

LCMS analysis was carried on Waters alliance 2695 HPLC system (Waters, Japan) contains $0.1-1500 \mu \mathrm{l}$ injectable auto-injector and is equipped with Waters ZQ Mass triple quadrupole Detector (model LAA 1369) and spectral intergradation were carried using Waters masslynx 4.2 software. The standard drugs Decitabine and Cedazuridine were obtained from Otsuka Pharmaceutical India Pvt Ltd., Ahmedabad, India. The internal standard drug Talazoparib was obtained from Pfizer Limited, Hyderabad, India. Milli Q water, methanol (HPLC grade), acetonitrile (HPLC grade), ammonium formate (Merck), formic acid (Merck), $0.45 \mu$ nylon membrane filter (Merck) were used in the study. Analyte-free and healthy human plasma sample was obtained from diagnostic laboratory, Guntur, AP.

\section{Preparation of standards and quality control (QC) samples}

The master stock solution of Decitabine and Cedazuridine at a concentration of $1 \mathrm{mg} / \mathrm{ml}(1000 \mu \mathrm{g} / \mathrm{ml})$ was prepared in methanol separately. The internal standard stock solution at a concentration of $100 \mathrm{ng} / \mathrm{ml}$ was prepared in methanol and all the prepared stock solutions were stored in a refrigerator at $2-8{ }^{\circ} \mathrm{C}$ until analysis. A master stock solution of Decitabine and Cedazuridine was added to the drug-free human plasma to obtain a concentration of $1,10,50$, 100,250 and $500 \mathrm{ng} / \mathrm{ml}$ and $100 \mathrm{ng} / \mathrm{ml}$ of internal standard. The aqueous calibration dilutions in the same concentration range were prepared using methanol as a diluent.

\section{Sample preparation}

Liquid-liquid extraction was carried for isolation of the drug and its internal standard from plasma. To $100 \mu \mathrm{l}$ Decitabine and Cedazuridine spiked standard or quality control plasma samples with different selected concentrations were added to $100 \mathrm{ng} / \mathrm{ml}$ of internal standard solution in a labeled polypropylene tube and vortexed. Then $2 \mathrm{ml}$ of methyl tertiary butyl ether was added, vortexed for $2 \mathrm{~min}$ and then the supernatant was organic layer is separated and evaporated under a stream of nitrogen and then reconstituted with methanol and acetonitrile in the ratio of 50: 50 $(\mathrm{v} / \mathrm{v})$ solvent as diluent. The reconstituted solution was used for method development and validation study.

\section{Method development}

Systematic trails of method development were performed to separate Decitabine, Cedazuridine and its internal standard Talazoparib. Method development trails were performed by progressively change in the method conditions and in each condition, the system suitability conditions such as tail factor, symmetric factor and resolution were verified and the conditions that produce best system suitability were considered for the further validation study.

\section{Method validation}

The developed method was validated as per available literature [12, 13] ICH [14] and FDA [15] guidelines and the parameters such as selectivity, sensitivity, accuracy, reproducibility, linearity and stability were studied in the validation.

\section{Sensitivity and standard/linearity curve}

Prior to the construction of the calibration curve, the limit of detection (LOD) and lower limit of quantification (LLOQ) for Decitabine and Cedazuridine analytes in the developed method was determined, and the standard curve for both the analystes was constructed from LLOQ and the concentration obtained as LLOQ was considered as the lowest concentration in the linear curve.

In the developed method, the calibration curve was prepared in the same biological matrix as the samples in the intended study by spiking the matrix with known concentrations of the analyte. Six concentrations including LLOQ for both the analytes and fixed known concentration of internal standard were prepared for construction of calibration range. The calibration curve was plotted by considering the concentration of individual analytes and the peak area response ratio of the analyte to the internal standard.

\section{Selectivity}

The interference of the blank plasma matrix in the separation of Decitabine, Cedazuridine and internal standard was determined in the selectivity study. In this study, blank plasma samples with no drug substance obtained from three different sources were analysed in the developed method and the chromatographic response of the analysis proves the selectivity of the method.

\section{Matrix effect}

Matrix effect for Decitabine, Cedazuridine and internal standard was evaluated by comparing the peak area ratio in the post-extracted plasma sample from 6 different drug-free blank plasma samples and neat reconstitution samples. The overall precision of the matrix factor is expressed as a coefficient of variation ( $\mathrm{CV} \%)$ and it should be $\leq 15 \%$ (CV \%).

\section{Precision, accuracy and recovery}

The Precise nature of the developed method was confirmed by measuring a minimum of six determinations in each concentration of both the analytes and containing known and fixed concentrations of internal standard. A minimum of three concentrations in the linearity range is studied in the precision study. The highest and medium concentrations in the linearity range considered as HQC and MQC were studied in the precision study along with LLOQ concentration. The coefficient of variation (CV) of each analyte in each concentration level should be $<15 \%$ except for the LLOQ, where it should be $<20 \%$. Further, precision is sub-divided in to within a run and inter-batch precision or repeatability which measures precision with time and may involve different analysts. In all the precision studies in each concentration level, the $\mathrm{CV}$ of both the analytes was calculated and confirms the repeatability and reproducibility of the developed method.

In recovery, the peak area response of the extracted analytes was compared with the un-extracted standards in the same concentration and the \% recovery was calculated. The $\%$ recovery of the analyte need not be $100 \%$, but the extent of recovery of an analyte and of the internal standard should be consistent, precise, and reproducible. Recovery was performed at HQC, MQC and LLOQ concentrations and the \% recovery in each recovery study was calculated and a \% recovery of less more than $85 \%$ was considered as the method is accurate except LLOQ where a \% recovery of more than $80 \%$ considered as acceptable.

\section{Stability}

Freeze and thaw stability, Short-term temperature stability and Long-term stability studies were performed for both the analytes containing fixed concentrations of internal standard in HQC, MQC and LLOQ concentrations. All stability determinations should use a set of samples prepared from a freshly made stock solution of the analyte in the appropriate analyte-free, interference-free biological matrix. Stock solutions of the analyte for stability evaluation should be prepared in an appropriate solvent at known concentrations.

\section{Freeze and thaw stability}

The stability of both the analytes in the developed method was determined after three freeze and thaw cycles. The samples stored at room temperature were thawed unassisted at room temperature and then refrozen under the same conditions and the same procedure was repeated for another two more cycles and then analysed after the completion of the third cycle. The \% stability of both the analytes was calculated by comparing the free and thaw stability study results of each analyte with the corresponding calibration curve results.

\section{Short-term temperature stability}

The samples were stored for $24 \mathrm{H}$ in room temperature and were analysed in the developed method. The \% stability of both the analytes was calculated and proved the short-term temperature stability of both the analytes in the developed method.

\section{Long-term stability}

The long-term stability was determined by storing the analytes at HQC, MQC and LLOQ concentrations under the same conditions as 
the study samples and were stored at $-30^{\circ} \mathrm{C}$. The sample volume was maintained such that it should be sufficient for analysis on three separate occasions. The concentrations of all the stability samples should be compared to the mean of back-calculated values for the standards at the appropriate concentrations from the first day of long-term stability testing. The \% stability was calculated and the long-term stability period of both the analytes in the developed method was determined from the results obtained in the long-term stability study.

\section{RESULTS}

Chromatographic conditions, especially the composition and nature of the mobile phase, were optimized through several trials to achieve better resolution and increase the signal of analytes in the study. A series of trials were conducted using different ratios of organic and $\mathrm{pH}$ modifiers and different buffer compositions having different $\mathrm{pH}$ to obtain the required separations. The results in each trail were reviewed and based on the results observed the optimised conditions for the separation and analysis of Decitabine and Cedazuridine were confirmed and the conditions were summarised in table 1.

In the optimised conditions, it eluted a peak with good characteristics for both Decitabine and Cedazuridine as well as Talazoparib (internal standard). The developed method gave a symmetric peak at a retention time of $1.7 \mathrm{~min}$ for Decitabine, $2.2 \mathrm{~min}$ for Cedazuridine and $3.5 \mathrm{~min}$ for Talazoparib and satisfied all the peak properties as per USP guidelines. The chromatogram of blank plasma matric shows no chromatographic detection at the retention time of analytes in the study and no other detections were observed confirms that the method was specific and suitable for the separation of Decitabine and Cedazuridine. The chromatogram of blank (3A), standard (3B), individual chromatograms of Decitabine (3C), Cedazuridine (3D) and internal standard (3E) in the developed method was given in fig. 3.

Table 1: Optimized method development parameters for decitabine and cedazuridine

\begin{tabular}{lll}
\hline S. No. & Condition & Result \\
\hline 1 & Column & Zorbax SB-CN $(4.6 \mathrm{~mm} \times 75 \mathrm{~mm}, 3.5 \mu \mathrm{m})$ column \\
2 & Pump mode & Isocratic \\
3 & Column temperature & Ambient \\
4 & Flow rate & $0.5 \mathrm{ml} / \mathrm{min}$ \\
5 & Injection volume & $10 \mu \mathrm{l}$ \\
6 & Run time & $5 \mathrm{~min}$ \\
7 & Detector & PDA and Mass spectrometry (MRM mode) \\
8 & Mobile phase pH & 4.9 \\
9 & Mobile phase & $0.1 \%$ ammonium formate and methanol in the ratio of $65: 45(\mathrm{v} / \mathrm{v})$ \\
\hline
\end{tabular}
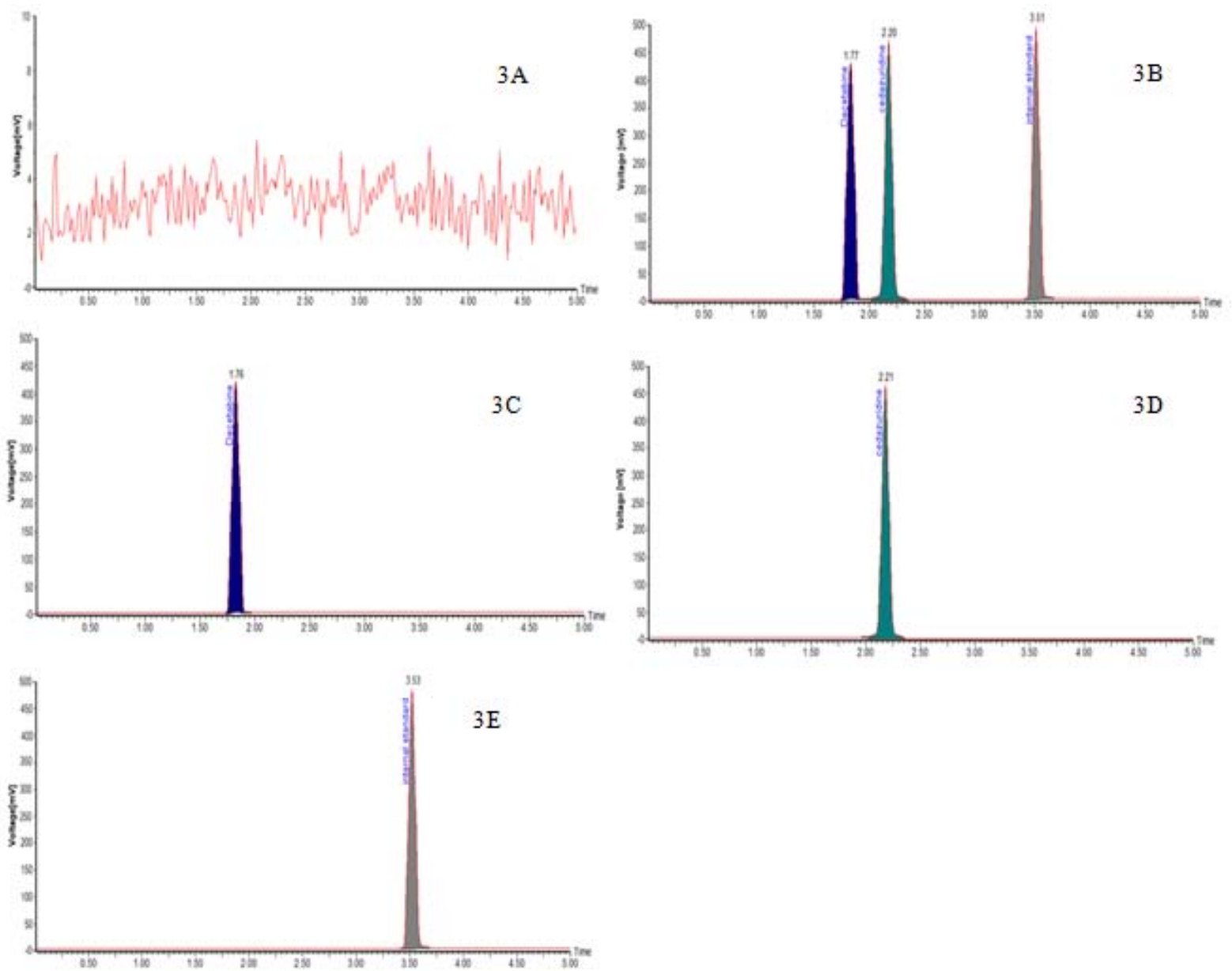

Fig. 3: Typical representative chromatograms chromatogram of blank plasma (3A), standard (3B), individual chromatograms of Decitabine (3C), Cedazuridine (3D) and internal standard (3E) 
The mass spectral characterization of separated analytes in the LC method was performed using mass detector operated at MRM (Multiple Reaction Monitoring) mode. Good response of product ions with better sensitivity was observed for both analytes and internal standard in positive ionization mode and hence detector was operated in positive ionization mode throughout the analysis.

The production spectrum of Decitabine shows characteristic fragment observed as $\mathrm{MH}^{+}$ion at $\mathrm{m} / \mathrm{z}$ of 229 corresponds to Decitabine and a characteristic fragment ion was identified as
$\mathrm{MH}^{+}$ion at $\mathrm{m} / \mathrm{z}$ of 114 with very high abundance. The product ion spectrum of Cedazuridine shows characteristic fragment observed as $\mathrm{MH}^{+}$ion at $\mathrm{m} / \mathrm{z}$ of 269 corresponds to Cedazuridine and a characteristic fragment ion was identified as $\mathrm{MH}^{+}$ion at $\mathrm{m} / \mathrm{z}$ of 118 with very high abundance. Whereas the characteristic fragment for internal standard was identified as $\mathrm{MH}^{+}$ion at $\mathrm{m} / \mathrm{z}$ of 381 and the high abundant characteristic fragment was identified at $\mathrm{m} / \mathrm{z}$ of 339 . The mass fragmentation spectra of Decitabine (4A), Cedazuridine (4B) and internal standard (4C) were given in fig. 4.
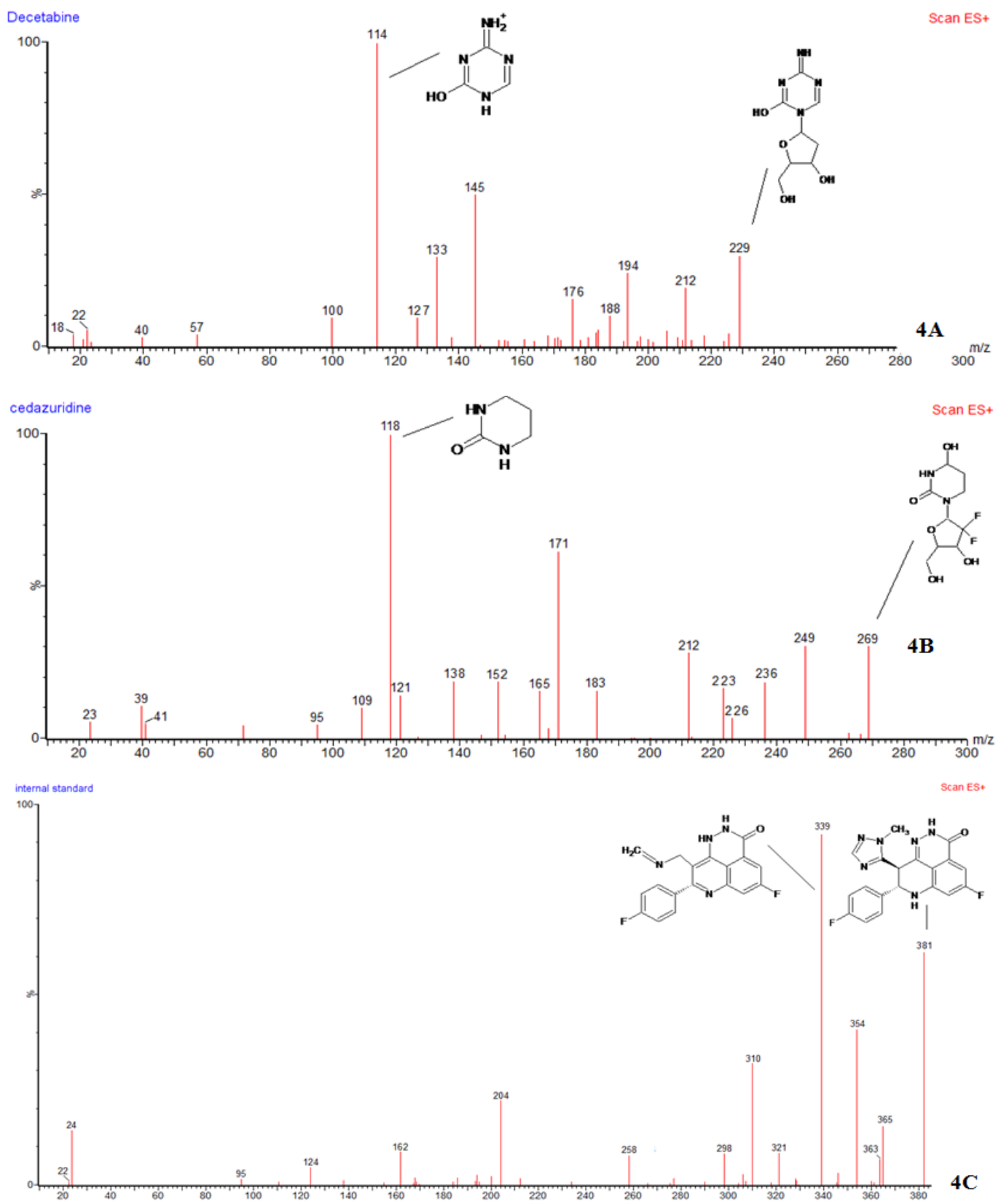

Fig. 4: Mass spectrum of decitabine (4A), Cedazuridine (4B) and internal standard (4C) showing parent and characteristic product ion in the developed method

The sensitivity (LOD) of Decitabine and Cedazuridine in the developed method was determined by the signal-noise ratio method for both the analytes and it was found that a very sensitive detection limit of $0.3 \pm 0.003 \mathrm{ng} / \mathrm{ml}$ was achieved confirms that the method is very sensitive and can detect up to a very low concentration of $0.1 \pm 0.010 \mathrm{ng} / \mathrm{ml}$. The LLOQ of both the analytes in the method was calculated and confirmed that $1.0 \mathrm{ng} / \mathrm{ml}$ as LLOQ. The calibration curve was constructed from LLOQ concentration for both the analytes.

The calibration curve for Decitabine and Cedazuridine in the developed method was constructed by least-square linear regression analysis of standard plots associated with six-point standard calibration curves. The calibration curves were plotted by 
considering the peak area ratio of standard and internal standard versus the concentration of standard prepared in the study. The calibration curve was linear within the concentration range of 1-500 $\mathrm{ng} / \mathrm{ml}$ for both Decitabine and Cedazuridine with constant proportionality and minimal data scattering. The regression equation was observed as $y=0.0053 x+0.0915\left(R^{2}=0.9997\right)$ for Decitabine and $\mathrm{y}=0.0061 \mathrm{x}+0.1313\left(\mathrm{R}^{2}=0.9993\right)$ for Cedazuridine. The linearity results were given in table 2 .

Table 2: Linearity results

\begin{tabular}{|c|c|c|c|c|c|c|}
\hline \multirow{2}{*}{$\begin{array}{l}\text { S. } \\
\text { No. }\end{array}$} & \multirow{2}{*}{$\begin{array}{l}\text { Concent-ration } \\
\text { in } \mu \mathrm{g} / \mathrm{ml}\end{array}$} & \multicolumn{3}{|l|}{ Peak area observed } & \multicolumn{2}{|c|}{ Peak area ratio of } \\
\hline & & Decitabine & Cedazuridine & Internal standard & Decitabine/IS & Cedazuridine/IS \\
\hline 1 & 1 & $22377.0 \pm 321.68$ & $31245.5 \pm 59.20$ & $310882.4 \pm 65.82$ & $0.072 \pm 0.00103$ & $0.101 \pm 0.00018$ \\
\hline 2 & 10 & $43226.3 \pm 36.54$ & $53640.3 \pm 146.32$ & $310262.9 \pm 249.70$ & $0.139 \pm 0.00001$ & $0.173 \pm 0.00041$ \\
\hline 3 & 50 & $115137.5 \pm 211.89$ & $138095.1 \pm 271.63$ & $310812.9 \pm 585.58$ & $0.370 \pm 0.00002$ & $0.444 \pm 0.00144$ \\
\hline 4 & 100 & $202878.7 \pm 597.56$ & $244934.4 \pm 609.63$ & $309585.3 \pm 850.65$ & $0.655 \pm 0.00035$ & $0.791 \pm 0.00299$ \\
\hline 5 & 250 & $441018.0 \pm 1136.34$ & $527056.6 \pm 1398.95$ & $311515.1 \pm 717.57$ & $1.416 \pm 0.00091$ & $1.692 \pm 0.00237$ \\
\hline 6 & 500 & $856465.8 \pm 1466.60$ & $985326.7 \pm 1713.93$ & $310470.9 \pm 373.54$ & $2.759 \pm 0.00142$ & $3.174 \pm 0.00296$ \\
\hline
\end{tabular}

Values given in the table are the average \pm standard deviation of three replicate experiments

In the precision and accuracy study, the back-calculated concentrations of the calibration points were found to be within the acceptable limit of less $15 \%$ in all the studied levels for both Decitabine and Cedazuridine. The \% RSD of the peak area ratios and the \% RSD of the amount estimated in the precision study for both the drugs Decitabine and Cedazuridine were found to be within the acceptable limit of less than 2 (table 3 ). The results confirm that the method was found to be precise and reproducible. The peak area ratio of the extracted plasma samples was compared with the aqueous analysis results of the same concentration level and the $\%$ recovery was calculated. A very high \% recovery of more than $90 \%$ was achieved for both the drugs in each concentration level. Hence high \% recoveries were observed for both Decitabine and Cedazuridine in the developed method.

Table 3: Summary results of validation parameters and stability studies

\begin{tabular}{|c|c|c|c|}
\hline \multirow[t]{2}{*}{ S. No. } & \multirow[t]{2}{*}{ Parameter } & \multicolumn{2}{|c|}{ Results observed } \\
\hline & & Decitabine & Cedazuridine \\
\hline \multirow[t]{4}{*}{1} & \% RSD in intraday Precision at & & \\
\hline & HQC & 0.11 & 0.40 \\
\hline & MQC & 1.12 & 0.59 \\
\hline & LLOQ & 1.05 & 1.46 \\
\hline \multirow[t]{4}{*}{2} & $\%$ RSD in inter-batch precision at & & \\
\hline & $\mathrm{HQC}$ & 0.50 & 0.59 \\
\hline & MQC & 1.11 & 0.63 \\
\hline & LLOQ & 0.90 & 0.41 \\
\hline \multirow[t]{4}{*}{3} & Amount estimated in Freeze and thaw stability at & & \\
\hline & $\mathrm{HQC}$ & 500.08 & 499.94 \\
\hline & MQC & 99.98 & 99.67 \\
\hline & LLOQ in $\mathrm{ng} / \mathrm{ml}$ & 0.980 & 0.998 \\
\hline \multirow[t]{4}{*}{4} & $\%$ RSD in Freeze and thaw stability at & & \\
\hline & $\mathrm{HQC}$ & 0.54 & 0.87 \\
\hline & MQC & 1.05 & 0.83 \\
\hline & LLOQ & 1.21 & 0.63 \\
\hline \multirow[t]{4}{*}{5} & Amount estimated in Short-term temperature stability at & & \\
\hline & $\mathrm{HQC}$ & 499.88 & 496.62 \\
\hline & MQC & 100.57 & 99.17 \\
\hline & LLOQ in $\mathrm{ng} / \mathrm{ml}$ & 0.982 & 0.996 \\
\hline \multirow[t]{4}{*}{6} & $\%$ RSD in Short-term temperature stability at & & \\
\hline & $\mathrm{HQC}$ & 0.75 & 0.60 \\
\hline & MQC & 0.84 & 1.05 \\
\hline & LLOQ & 1.02 & 0.67 \\
\hline \multirow[t]{4}{*}{7} & Amount estimated in Long-term stability at & & \\
\hline & $\mathrm{HQC}$ & 500.16 & 496.70 \\
\hline & MQC & 100.02 & 99.52 \\
\hline & LLOQ in $\mathrm{ng} / \mathrm{ml}$ & 0.980 & 0.994 \\
\hline \multirow[t]{4}{*}{8} & $\%$ RSD in Freeze-thaw stability at & & \\
\hline & $\mathrm{HQC}$ & 0.82 & 0.90 \\
\hline & MQC & 1.43 & 1.17 \\
\hline & LLOQ & 1.19 & 0.62 \\
\hline
\end{tabular}

The results given in the table are the average of six replicate experiments

There is considerable change in the peak area ratios of Decitabine and Cedazuridine with the internal standard when the drug was exposed to three freeze-thaw cycles and kept in room temperature for $24 \mathrm{H}$ was observed in freeze-thaw stability and short term stability respectively. There is also no significant change in the results were observed when the analytes were stored at $-30{ }^{\circ} \mathrm{C}$ for $30 \mathrm{~d}$ in long-term stability. In all the three stability studies, the peak corresponds to Decitabine and Cedazuridine along with the internal standard was observed and clear base-line with no other detections were observed in the chromatogram and clear mass fragmentation corresponds to 
analytes studied was observed. The \% RSD of the peak area ratios was within the acceptable limit of less than 2 and the back-calculated concentrations in each concentration level in all the stability studies were very close to the standard. The results of the stability studies (table 3) confirms that the method was found to be stable.

\section{DISCUSSION}

LC-MS/MS is one of the most powerful analytical tools in clinical pharmacokinetics for its selectivity, sensitivity and reproducibility. The present study aimed to develop a simple and novel LCMS method for the simultaneous estimation of Decitabine and Cedazuridine in spiked human plasma. The survey of the available analytical methods for the analysis of Decitabine and Cedazuridine proves that there is a need for LCMS analytical method for analysis of these drugs in biological samples.

A simple and convenient liquid-liquid extraction technique was utilized for the extraction of Decitabine and Cedazuridine from the plasma samples along with internal standards in the study. Different solvents such as chloroform, diethyl ether, acetone, ethyl acetate etc were studied for extraction of the analytes in the spiked plasma. The high extraction efficiency was achieved using the solvent methyl tertiary butyl ether and hence is used as a suitable solvent for extraction of analytes from plasma.

The mass spectral characterization of both the analytes along with internal standard was carried on positive ion mode and characteristic fragments were identified in the spectra. The method was validated in terms of linearity, precision, recovery and stability studies like short term, long term and freeze-thaw stability studies.

A very sensitive detection limit of $0.3 \pm 0.003 \mathrm{ng} / \mathrm{ml}$ and quantification limit of $0.1 \pm 0.010 \mathrm{ng} / \mathrm{ml}$ was achieved proves that the method is sensitive and the calibration curve was constructed from LOQ to a very high concentration of $500 \mathrm{ng} / \mathrm{ml}$ for both the analytes confirms that the analytical range was very broad and sensitive. Hence confirms that the method is having high applicability. In the developed method, the other validation parameters were found to be acceptable and confirm that the method can suitable for the simultaneous analysis of Decitabine and Cedazuridine in human plasma.

The findings in the present study were compared with the available analytical methods for the simultaneous analysis of Decitabine and Cedazuridine. The method reported by Sunilkumar et al., 2014 [10] and Donthineni et al., 2014 [11] were reported for the analysis of Decitabine in formulations. There is no analytical method reported for the simultaneous estimation of Decitabine and Cedazuridine in formulations and in biological samples. Hence the method reported in the present study will be the best choice for the simultaneous analysis of Decitabine and Cedazuridine in human plasma samples and may be applicable for the analysis of drugs in bioequivalence studies.

\section{CONCLUSION}

In conclusion, the LC-MS/MS method for the simultaneous quantization of Decitabine and Cedazuridine in human plasma was developed and fully validated as per FDA guidelines. This method offers significant advantages over those previously reported, in terms of improved sensitivity and selectivity, the faster run time (5 min) and lower sample requirements. Thus the volume of samples to be collected per time point from an individual during trial is reduced significantly, allowing the inclusion of additional points. The method having very sensitive with a lower limit of quantification of $1 \mathrm{ng} / \mathrm{ml}$ and having extended up to a very high upper quantification limit of $500 \mathrm{ng} / \mathrm{ml}$. The current method has high recoveries, acceptable precision and adequate sensitivity for the simultaneous quantization of Decitabine and Cedazuridine in human plasma samples obtained for pharmacokinetic, bioavailability or bioequivalence studies.

\section{FUNDING}

The authors declare that we have "no funding support for this study"

\section{AUTHORS CONTRIBUTIONS}

All authors have contributed equally.

\section{CONFLICT OF INTERESTS}

The authors declare that we have no conflict of interest.

\section{REFERENCES}

1. Christine BY, Peter AJ. Epigenetic therapy of cancer: past, present and future. Nat Rev Drug Discovery 2006;5:37-50.

2. Li LH, Olin EJ, Fraser TJ, Bhuyan BK. Phase specificity of 5azacytidine against mammalian cells in tissue culture. Cancer Res 1970;30:2770-5.

3. Stanton LG, Paolo FC, Basem MW, Richard JC. Chapter 57pharmacology and molecular mechanisms of antineoplastic agents for hematologic malignancies, hematology (Seventh Edition), Elsevier Inc; 2018. p. 849-912.

4. Michael RS, Olatoyosi 0, Philip CA. An oral fixed-dose combination of decitabine and cedazuridine in myelodysplastic syndromes: a multicentre, open-label, dose-escalation, phase 1 study. Lancet Haematol 2019;6:194-203.

5. Guillermo GM, Elizabeth AG, David PS. Oral cedazuridine/decitabine for MDS and CMML: a phase 2 pharmacokinetic/pharmacodynamic randomized crossover study. Blood 2020;136:674-83.

6. Jesus DG, Samarpana C, Amit V, Aditi S. The evolution of epigenetic therapy in myelodysplastic syndromes and acute myeloid leukemia. Semin Hematol 2021;58:56-65.

7. Mohammed IB, Siva SR, Venu S, Sreenivasulu M, Vanitha PK. RP-HPLC-PDA method development, validation and stability studies of the novel antineoplastic drug combinationdecitabine and cedazuridine. J Pharm Res Int 2020;32:10-6.

8. Glory H, Teja BB, Ashok KK, Ravindra RY. Stability indicating RP-HPLC method development and validation of decitabine drug in the formulation. Int J Pharmtech Res 2011;3:237-43.

9. Yub Raj N, Manish Srivastava, Nafees Ahmad, Kriti Soni, Kanchan Kohli. Stability indicating RP-HPLC method for the estimation of decitabine in bulk drug and lipid-based nanoparticles. Int J Pharm Sci 2014;5:294-302.

10. Sunilkumar Adupa, Satish K, Ravi J. Development and validation method for decitabine injection by RP-HPLC. Int J Pharm Sci Res 2014;5:3425-9.

11. Donthineni Kalyan, Swetha A, Arabinda Patnaik, Om Prakash Chary V. A RP-HPLC method development and validation for estimating decitabine with its stability studies. Int J Innov 2014;2:1495-506.

12. Yahdiana Harahap, Norma Andriyani, Harmita. Method development and validation of lercanidipine in human plasma by liquid chromatography-tandem mass spectrometry. Int J Appl Pharm 2018;10:87-91.

13. Prasad PBN, Satyanarayana K, Krishna Mohan G. Simultaneous determination of metformin, linagliptin in jentadueto and metformin, saxagliptin in kombiglyze by LC-MS method. Int J Pharm Pharm Sci 2018;10:110-1.

14. Validation of Analytical Procedures: Text and Methodology, Q R1, Step 4, ICH Tripartie Guidelines; 2005.

15. Validation of Compendial Methods, United States Pharmacopoeia 30, National Formulary 25, The United States Pharmacopoeial Convention, Inc., Rockville, Md, USA; 2007. 